\title{
SCHUR-CONVEXITY, SCHUR GEOMETRIC AND SCHUR HARMONIC CONVEXITIES OF DUAL FORM OF A CLASS SYMMETRIC FUNCTIONS
}

\author{
HUAN-NAN SHI AND JING ZHANG
}

\begin{abstract}
By the properties of Schur-convex function, Schur geometrically convex function and Schur harmonically convex function, Schur-convexity, Schur geometric and Schur harmonic convexities of the dual form for a class of symmetric functions are simply proved. As an application, several inequalities are obtained, some of which extend the known ones.
\end{abstract}

Mathematics subject classification (2010): 26D15, 05E05, 26B25.

Keywords and phrases: Schur-convexity, Schur geometric convexity, Schur harmonic convexity, inequality, log-convex function, symmetric functions, dual form.

\section{REFERENCES}

[1] Y.-M. Chu And Y.-P. Lv, The Schur harmonic convexity of the Hamy symmetric function and its applications, Journal of Inequalities and Applications, 2009, Article ID 838529 (2009), 10 pages.

[2] Y. M. CHU AND T. C. SUN, The Schur harmonic convexity for a class of symmetric functions, Acta Mathematica Scientia, 30B, 5 (2010), 1501-1506.

[3] Y.-M. ChU, G.-D. WANG AND X.-H. ZHANG, The Schur multiplicative and harmonic convexities of the complete symmetric function, Mathematische Nachrichten, 284, 5-6 (2011), 653-663.

[4] Y.-M. CHU AND W.-F. XIA, Solution of an open problem for Schur convexity or concavity of the Gini mean values, Science in China Series A: Mathematics, 52, 10 (2009), 2099-2106.

[5] Y.-M. ChU, W.-F. XIA AND X.-H. ZHANG, The Schur concavity, Schur multiplicative and harmonic convexities of the second dual form of the Hamy symmetric function with applications, Journal of Multivariate Analysis, 105, 1 (2012), 412-421.

[6] Y. M. Chu, X. M. Zhang AND G. D. WANG, The Schur geometrical convexity of the extended mean values, Journal of Convex Analysis, 15, 4 (2008), 707-718.

[7] K. Z. GUAN, A class of symmetric functions for multiplicatively convex function, Mathematical Inequalities \& Applications, 10, 4 (2007), 745-753.

[8] W. D. JiAnG, Some properties of dual form of the Hamy's symmetric function, Journal of Mathematical Inequalities, 1, 1 (2007), 117-125.

[9] A. W. Marshall, I. OlKin AND B. C. ARNOLD, Inequalities: Theory of Majorization and Its Application (Second Edition), Springer, New York, 2011.

[10] I. RovEnŢA, Schur convexity of a class of symmetric functions, Annals of the University of Craiova, Mathematics and Computer Science Series, 37, 1 (2010), 12-18.

[11] H. N. SHI, Theory of Majorization and Analytic Inequalities, Harbin Institute of Technology Press, Harbin, 2012. (in Chinese)

[12] H.-N. SHI AND J. ZHANG, Schur-convexity of dual form of some symmetric functions, Journal of Inequalities and Applications, 2013, Article 295 (2013), 9 pages.

[13] T.-C. Sun, Y.-P. Lv AND Y.-M. Chu, Schur multiplicative and harmonic convexities of generalized Heronian mean in $n$ variables and their applications, International Journal of Pure and Applied Mathematics, 55, 1 (2009), 25-33.

[14] B. Y. WANG, Foundations of Majorization Inequalities, Beijing Normal University Press, Beijing, 1990. (in Chinese) 
[15] S. H. WANG, T. Y. ZhANG AND Z. Q. HUA, Schur convexity and Schur multiplicatively convexity and Schur harmonic convexity for a class of symmetric functions, Journal of Inner Mongolia University for the Nationalities (Natural Sciences), 26, 4 (2011), 387-390. (in Chinese)

[16] K. Watanabe, On relation between a Schur, Hardy-Littlewood-Pólya and Karamata's theorem and an inequality of some products of $x^{p}-1$ derived from the Furuta inequality, Journal of Inequalities and Applications, 2013, Article 137 (2013), 7 pages.

[17] W.-F. XIA AND Y.-M. CHU, The Schur convexity of the weighted generalized logarithmic mean values according to harmonic mean, International Journal of Modern Mathematics, 4, 3 (2009), 225233.

[18] W.-F. XIA AND Y.-M. ChU, The Schur multiplicative convexity of the weighted generalized logarithmic mean in $n$ variables, International Mathematical Forum, 4, 25 (2009), 1229-1236.

[19] W.-F. XIA AND Y.-M. CHU, The Schur harmonic convexity of Lehmer means, International Mathematical Forum, 4, 41 (2009), 2009-2015.

[20] W. F. XIA AND Y. M. CHU, Schur-convexity for a class of symmetric functions and its applications, Journal of Inequalities and Applications, 2009, Article ID 493759 (2009), 15 pages.

[21] W.-F. XIA AND Y.-M. ChU, The Schur convexity of Gini mean values in the sense of harmonic mean, Acta Mathematica Scientia, 31B, 3 (2011), 1103-1112.

[22] W.-F. XIA, G.-D. WANG AND Y.-M. CHU, Schur convexity and inequalities for a class of symmetric functions, International Journal of Pure and Applied Mathematics, 58, 4 (2010), 435-452.

[23] X. M. Zhang, Geometrically Convex Functions, An'hui University Press, Hefei, 2004. (in Chinese)

[24] X.-M. Zhang AND Y.-M. ChU, Convexity of the integral arithmetic mean of a convex function, Rocky Mountain Journal of Mathematics, 40, 3 (2010), 1061-1068. 